\title{
Reflective Abstraction of Junior High School Students in Reconstructing The Factorization Concept
}

\author{
Helmi Rahmawati, I Ketut Budayasa, and Rooselyna Ekawati \\ Postgraduate Program, Universitas Negeri Surabaya, INDONESIA \\ helmirahmawati16070785052@mhs.unesa.ac.id, ketutbudayasa@unesa.ac.id, rooselynaekawati@unesa.ac.id
}

\begin{abstract}
Construction of the mathematical concept is important to support students' success in learning. Factorization is one of the important subjects to be mastered by students in order to solve advanced mathematics problems. Reflective abstraction is a mental mechanism which someone can build all mathematical concepts at all levels. This study used the mental structure of APOS theory (Action, Process, Object, Schema) to describe the mental mechanisms of interiorization, encapsulation, and thematization. This study aims to reveal and describe the reflective abstraction of students in reconstructing the factorization concept. The results obtained that female student at the action stage connected the mathematics knowledge that belongs to the factorization content to find the answer. In the process stage, she could explain how she got the answer. In the object stage, she could explain the rules used. In scheme stage, she could connected the actions, processes, objects, and schemes that have been formed to form a factorization scheme and she could do more complex tasks, while male student only reach the process phase. Therefore, the reflective abstraction of female student is better than male student with medium mathematics ability in reconstructing the factorization concept.
\end{abstract}

Keywords- Reflective Abstraction, Reconstruction of Concept, Factorization.

\section{INTRODUCTION}

Factorization is one of the materials that included in algebra knowledge which is important to be mastered by students in order to understand advanced mathematics. According to [1] understanding algebra is a key for success in future mathematics courses, including geometry and calculus. The materials associated with factorization are equation and innequality of quadratic. In order to understand the related materials, the students must first be able to factorize the algebraic form. Therefore, it is necessary to instill the concept of factorization in students themselves in order to understand other related material easily. This is because understanding the concept is important for supporting the success of students in learning.

Concepts are the basic building blocks for thinking and communicating [2]. One of the efforts in building the concept of mathematics is through abstraction. This is in line with Skemp's opinion that concepts were formed from the process of abstraction and classification, where abstraction is the activity of thinking consciously of the similarities of objects between experiences. While classification is a grouping of experiences that have similarities from the results of abstraction [3].

There are three forms of abstraction which are; the empirical abstraction where the focus is on the objects properties and knowledge on the object derives from his own properties, the pseudo-empirical abstraction where the focus is on actions which leaves out the properties that the action of the subjects have introduced into objects and reflective abstraction where further constructions can then be accomplished by its existing structures used to construct new structures [4].

This study discusses about reflective abstraction, where reflective abstraction consists of a system of action or operation at a lower level, certain characteristics that reflect on the action or operation at the higher level it assures. Awaring the previous construction process is done through reconstruction in the new field, then proceed with the reconstruction which transcend by integrating the previous construction. In other words, reflective abstraction has two components that reflecting the operations at a lower level and reconstructing and then integrating them to a higher level [5].

There are six kinds of reflective abstraction in mathematics education: interiorization, coordination, reversal, encapsulation, thematization, and generalization. The mechanism of reflective abstraction can be applied to mental structures, namely, action, process, object, and schema, to construct new mental structures [6]. Therefore, this study aims to reveal and describe the reflective abstraction of students in reconstructing the factorization concept using the mental structure of APOS theory to describe the mental mechanisms of interiorization, encapsulation, and thematization.

This research aims to reveal and describe the reflective abstraction of junior high school students in reconstructing the factorization concept based on gender differences. 


\section{METHOD}

This study used a qualitatively descriptive approach that aims to reveal and describe the reflective abstraction of junior high school students in reconstructing the concept of factorization. The subject participant of this research is one male and one female seventh graders in a junior high school in Surabaya with medium mathematics ability. The subjects were selected based on the results of factorization tests were used to see students' mathematics abilities. The subject selection was also conducted by considering the documentation record of the students' mathematics' performance on the first mid semester. Students with medium score on factoring test and first mid semester are selected as research subjects. The data of this study is about students' reflective abstraction, were collected through giving test and interview based on the reconstruction test of factorization. The data obtained were analyzed based on students' reflective abstraction.

The reflective abstraction in this study is an abstraction involving reflection, in the sense of realizing and contemplating operations performed from the cognitive level or lower stage to the higher stage which refers to the mental structure of APOS Theory: action, process, object and scheme that has aims to describe the mental mechanisms of interiorization, encapsulation and thematization of students in reconstructing mathematical concepts. The reflective abstraction indicators in this study can be seen in Table 1 .

TABLE I. REFLECTIVE ABSTRACTION INDICATORS

\begin{tabular}{|c|c|}
\hline \multicolumn{2}{|r|}{ Reflective Abstraction } \\
\hline Stages & Indicators \\
\hline Action & $\begin{array}{l}\text { - Connecting the mathematics knowledge that } \\
\text { belongs to the factorization content to find the } \\
\text { answers. }\end{array}$ \\
\hline Process & $\begin{array}{l}\text { - Connecting more mathematics knowledge related to } \\
\text { the factorization content to find the answers. } \\
\text { - Explain how the answers were obtained, }\end{array}$ \\
\hline Objects & $\begin{array}{l}\text { - Connecting the mathematics knowledge with the } \\
\text { factorization knowledge which were wholly owned. } \\
\text { - Awaring of the total process by realizing the } \\
\text { transformation done and able to explain the rules } \\
\text { used in every step of the settlement done }\end{array}$ \\
\hline Schema & $\begin{array}{l}\text { - Connecting actions, processes, and objects, and } \\
\text { leads to formation of a factorization scheme. } \\
\text { - Using schemes that have been formed through } \\
\text { actions, processes, objects that in mind to form new } \\
\text { objects that can be used in accomplishing more } \\
\text { complex tasks. }\end{array}$ \\
\hline
\end{tabular}

\section{RESULTS AND DISCUSSION}

Based on the research questions, the findings in this study relate to students' reflective abstraction in reconstructing the concept of factoring described briefly. The assigned tasks used in this study are as follows:

What are the factors of $8 p^{2}+7 p q-15 q^{2}$ ?

\section{A. Reflective Abstraction of Male Student (MS)}

Based on Figure 1, it can be seen that male student's answer the problem correctly begins with looking for factors of 120 . Then in the first step replaces the middle term with factors which, if multiplied produce 120, if adds up produce 7 . In the second step student classify term into two parts. In the third step student look for factors from the two terms, and in the fourth step student multiply the common factor with other factors.

$$
\begin{aligned}
& 8 p^{2}+7 p q-15 q^{2} \\
& =8 p^{2}-8 p q+15 p q-15 q^{2} \\
& =\left(8 p^{2}-8 p q\right)+\left(15 p q-15 q^{2}\right) \\
& 8 p(p-q)+15 q(p-q) \\
& (p-q)(8 p+15 q)
\end{aligned}
$$
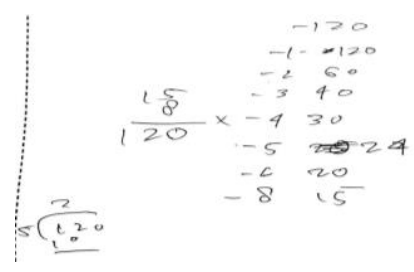

Figure 1: Male student's answer

In action stage, MS could define the definition of factorization and find the coefficients, variables, constants, and terms of the question. He could connect the mathematics knowledge that belongs to the factorization content to find the answers, as could mention what knowledge was required in factoring the algebraic form. According to [7] a transformation is first conceived as an action, when it is a reaction to stimuli which an individual perceives as external. It consists in following specific external or internal instructions, and the need to perform each step of the transformation explicitly.

In process stage, MS could connect more mathematics knowledge related to the factorization to find the answers. He also explained his strategy and reason in interview session. An important characteristic of a process shows that MS is able to describe, or reflect upon, the steps of the transformation wholly in her/his mind without actually performing those steps [8]. In this case, he could choose the factors of 120 which, when it is added can produce 7 . He can imagine and give the reason if the sign of the factors were changes could not get the value $b$, by saying the results are different. but if the factors were switched its place would produce the same value. In objects stage, MS had not been able to relate the mathematics knowledge to the factorization knowledge that they have, he could not explain the rules used in the completion steps that he was taken. In schema stage, MS could not connect the actions, processes, and objects, and leads to formation of a factorization scheme. MS do not know how to make sure whether the answer is right or wrong. The following below is the interview transcript of Male Subject (MS).

\section{Action Stage.}

$\begin{array}{ll}\mathrm{R}: & \text { What is factorization? } \\ \mathrm{MS}: & \text { Factorization is combining algebra forms to } \\ \text { obtain multiplication form }\end{array}$


terms in the algebra form of the question!

MS : $\quad$ Coefficients are 8, 7 and -15

Variables are $p^{2}, p q$ and $q^{2}$

There is no the constants in the algebra form of the question

The terms of algebra form are $8 p^{2}, 7 p q$ and $15 q^{2}$

$\mathrm{R}$ : According to you, what knowledge we use in factoring algebra form?

MS : $\quad$ Multiplication and division

\section{Prosess Stage}

$\mathrm{R}$ : Please explain how you got the answer

MS : The first step is multiplying 8 by -15 . After we have the answer is -120 , we have to find the factors of -120 . Then, we have to choose the factors which if added yielding 7 . So, I replacing the initial middle term with the selected factors. In this case, replacing $7 p q$ with $-8 p q$ and $15 p q$. After that, brackets the first two terms and the last two term. Thereafter, the first and last two terms was factored. So I have obtained the common factor in both. So I have obtained the factors of $8 p^{2}+7 p q-15 q^{2}$ were $(p-q)$ and $(8 p+15 q)$.

$\mathrm{R}$ : I have seen that you put negative sign in 8 , if I change the sign of the factors which 8 is positive and 15 is negative, could it be?

MS : It couldn't

$\mathrm{R}$ : Why?

MS : $\quad$ The result is different

$\mathrm{R}$ : If the factor I switched its place, could it be?

MS : Yes

R : Why?

MS : $\quad$ Because although be reversed the results were same

$\mathrm{R}$ : What is it called?

MS : Commutative principle

$\mathrm{R}$ : In addition to commutative principle, what properties do you know?

MS : Associative and distributive principles

\section{Objects Stage}

$\mathrm{R}$ : At the first step, what did you do?

MS : describes the middle term

$\mathrm{R}$ : Why you choose it step?

MS : to simplify it

$\mathrm{R}$ : At the second step, what did you do?

MS : $\quad$ Separating the tribes with brackets

$\mathrm{R}$ : What rules did you used?

MS : Maybe.. Associative?

$\mathrm{R}$ : What rules did you used at the third step?

MS : Mmmm.... I don't know.

$\mathrm{R}$ : What rules did you used at the fourth step?

FS : Associative

\section{Schema Stage}

$\mathrm{R}$ : If the question is "Determine the factors of $36 k^{2}+9 k g-7 g^{2}$ ?", what are the factors?
MS

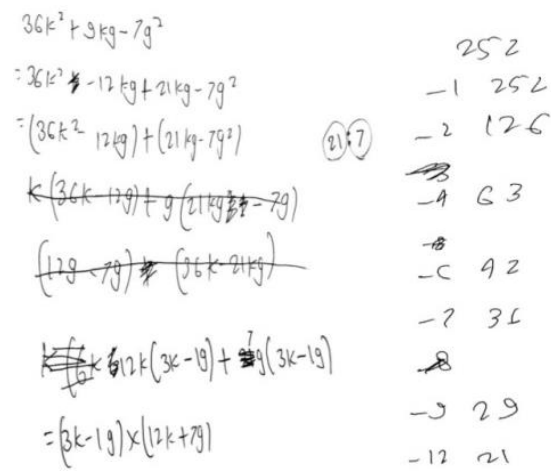

$\mathrm{R}$ : How do you convince yourself that your answer is correct?

MS : I feel confident with my answer

\section{B. Reflective abstraction of Female Student (FS)}

Based on Figure 2, it can be seen that female student's answer correctly which begins by looking for factors of 120 . Then in the first step replaces the middle term with factors which, if multiplied produce 120 , if adds up produce 7 . In the second step student classify sub term into two parts. In the third step student look for factors from the two terms, and in the fourth step student multiply the common factor with other factors.

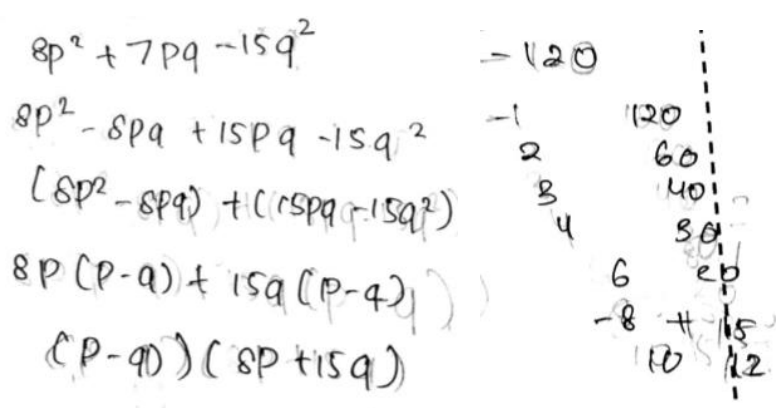

Figure 2: Female student's answer

In action stage, FS could define the definition of factorization, she could find the coefficients, variables, constants, and terms of the question. She could connect the mathematics knowledge that belongs to the factorization content to find the answers, as could mention what knowledge was required in factoring the algebraic form. A mathematical concept is first understood as an action; a set of step-by-step instructions performed explicitly to transform physical or mental objects [5].

In process stage, FS could connect more mathematics knowledge related to the factorization content to find the answers and explain how she got the answer which can be seen on interview transcripts. She could choose the factors of 120 which, when added, produce 7 . He can imagine and give the reason if the sign of the factors were changes could not get the value $b$, by saying the results are different. but if the factors were switched its place would produce the same value. 
The student has interiorized an action into a process if she/he can perform an internal operation that is done (or imagined) essentially the same transformation entirely in the mind, without the need to perform all the specific steps [9].

In objects stage, FS had been able to relate the mathematics knowledge to the factorization knowledge that they have entirety, she could explain the rules used in the completion steps that he was taken such as using associative and distributive principles. If one becomes aware of the total process, realizes that transformations can act on that totality and can actually constructs such transformations (explicitly or in one's imagination), it can be determined that he encapsulated the process into a cognitive object [10].

In schema stage, FS could connect actions, processes, and objects, and leads to formation of a factorization scheme. She has used the schemes that have been formed through actions, processes, objects that in mind to form new objects that can be used in accomplishing more complex tasks. This is can be inferred from when students could answer the question that have given. She could apply the factorization scheme that she has for more difficult questions. But she has not been able to determine the factors for common algebra forms such as $a x^{2}+b x y+c y^{2}$. In this stage FS known how to make sure whether the answer is right or wrong. Scheme is a coherent system of actions, processes, objects and other schemes that have existed in the minds of students used to confront situations of certain mathematical problems. The coherence of a schema is what allows one to decide if it can be used in a particular mathematical situation [5]. Thematization is the mechanism involved in constructing an object from a schema [8]. The interview transcript of Female Subject (FS) was described.

\section{Action Stage.}

\section{$\mathrm{R}$ : What is factorization?}

FS : Factorization is operation that uses division operation to produce multiplication of algebra terms

$\mathrm{R}$ : Find the coefficients, variables, constants, and terms in the algebra form of the question

FS : $\quad$ Coefficients are 8,7 and -15 Variables are $p^{2}, p q$ and $q^{2}$

There is no the constants in the algebra form of the question

The terms of algebra form are $8 p^{2}, 7 p q$ and $15 q^{2}$

$\mathrm{R}$ : According to you, what knowledge we use in factoring algebra form?

FS : Multiplication and division

\section{Prosess Stage}

$\mathrm{R}$ : Please explain how you got the answer

FS : First is to look for factors of -120 which is obtained after multiplying 8 by -15 . After that, choose the factors which if added yielding 7 . Then, replace $7 p q$ with $-8 p q+15 p q$. So, brackets the first two terms and the last two term. The next is to look for the factors of $\left(8 p^{2}-8 p q\right)$ and $\left(15 p q-15 q^{2}\right)$. So I have obtained the common factor, that is $(p-q)$.

So I have obtained the factors of $8 p^{2}+7 p q-15 q^{2} \quad$ were $\quad(p-q) \quad$ and $(8 p+15 q)$

$\mathrm{R}$ : I have seen that you put negative sign in 8 , if I change the sign of the factors which 8 is positive and $\mathbf{1 5}$ is negative, could it be?

FS : It couldn't

$\mathrm{R}$ : Why?

FS: The result was changed to -7 , it's does not produce the middle term.

$\mathrm{R}$ : If the factor I switched its place, could it be?

FS : Yes

R : Why?

FS : Because although be reversed the results were same

$\mathrm{R}$ : What is it called?

FS : $\quad$ Commutative principle

$\mathrm{R}$ : In addition to commutative principle, what properties do you know?

FS : $\quad$ Associative and distributive principles

\section{Objects Stage}

$\mathrm{R}$ : At the first step, what did you done?

FS : describes the middle term

$\mathrm{R}$ : Why you choose it step?

FS : to simplify it

$\mathrm{R}$ : At the second step, what did you do?

FS : Separating the terms with brackets

$\mathrm{R}$ : What rules did you used?

FS : mmm.. wait a minute, Asosiative

$\mathrm{R}$ : What rules did you used at the third step?

FS : Distributive.

$\mathrm{R}$ : What rules did you used at the fourth step?

FS : Distributive

\section{Schema Stage}

$\mathrm{R}$ : If the question is "Determine the factors of $36 k^{2}+9 k g-7 g^{2}$ ?", what are the factors?

FS :
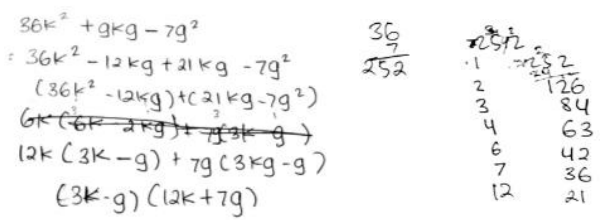

$\mathrm{R}$ : How do you convince yourself that your answer is correct?

FS : $\quad$ By multiplying the factors that I have obtained so that it returns to the algebra form which that we looking for its factors. 


\section{CONCLUSION}

Based on the results and discussion above, female student was able to perform a reflective abstraction from the lower stage to the higher stage, ie from action to process, process to object, object to schema, although not to the higher stage of the scheme. Therefore, female student could describe the mental mechanisms of interiorization, encapsulation and thematization in reconstructing of the factoring concept. While male student only capable performing a reflective abstraction up to the process stage. So that male student only able to describe the mental mechanisms of the interiorization in reconstructing of the factorization concept. So it can be concluded that the reflective abstraction of female student is better than that of male student with medium mathematics ability.

\section{ACKNOWLEDGEMENT}

The authors thank to the Junior High School 36 Surabaya for helping and supporting this study and special thank to all students and teachers who actively involved in this study

\section{REFERENCES}

[1] J. R. Star, A. Foegen, M. R. Larson, W. G. McCallum, J. Porath, and R. M. Zbiek, Teaching Strategies for Improving Algebra Knowledge in
Middle and High School Students. NCEE 2014-4333). Washington, DC: National Center for Education Evaluation and Regional Assistance (NCEE), Institute of Education Sciences, U.S. Department of Education, 2015, pp. 1-64.

[2] R. Arends, Learning to Teach. (5th Edition), Singapore: The McGrawHill Companies, 2001

[3] R. Skemp, Psychology of Learning Mathematics, New Jersey: Lawrence Erlbaum Associates, Inc, 1987.

[4] S. Kërënxhi and P. Gjoci, "Involvement of Algebraic-Geometrica Duality in Shaping Fraction's Meaning and Calculation Strategies with Fractions", Journal of Educational and Social Research, vol. 7, No.1, pp. 151-157, 2017.

[5] I. Cetin and E. Dubinsky, "Reflective abstraction in computational thinking", Journal of Mathematical Behavior, vol. 47, pp. 70-80, 2017.

[6] I. Cetin, "Students' Understanding of Loops and Nested Loops in Computer Programming: An APOS Theory Perspective", Canadian Journal of Science, Mathematics and Technology Education, vol. 15, no. 2, pp. 155-170, 2015

[7] S. Bansilal, D. Brijlall, and M. Trigueros, "An APOS study on preservice teachers' understanding of injections and surjections", Journal of Mathematical Behavior, vol. 48, pp. 22-37, 2017.

[8] V. F. Moll, M. Trigueros, E. Badillo, and N. Rubio, "Mathematica objects through the lens of two different theoretical perspectives: APOS and OSA, Educ Stud Math, vol. 91, pp. 107-122, 2016

[9] I. García-Martínez and M. Parraguez, "The basis step in the construction of the principle of mathematical induction based on APOS theory", Journal of Mathematical Behavior, vol. 46, pp. 128-143, 2017.

[10] I. Arnon, J. Cottrill, E. Dubinsky, A. Oktac, S. R. Fuentes, M. Trigueros, and K. Weller, APOS Theory: A Framework for Research and Curriculum Development in Mathematics Education. New York: Springer Science+Business Media, 2017 